\title{
Validation of Improved Dynamic Spectrum and Traffic Management Models of Internet Pricing of Fair DSL-LTE Multiple QoS Network
}

\author{
Fitri Maya Puspita ${ }^{1 *}$, Syalia Arda', Robinson Sitepu', Yunita ${ }^{1}$, Evi Yuliza', Sisca Octarina', Sugandi Yahdin ${ }^{1}$ \\ ${ }^{1}$ Department of Mathematics, Faculty of Mathematics and Natural Sciences, Sriwijaya University, Palembang, 30862, Indonesia \\ *Corresponding author: fitrimayapusita@unsri.ac.id
}

\begin{abstract}
Currently, the development of communication technology is very rapid, especially on the internet. Therefore, with the increasing need for the internet, the quality of service must also be improved. This study aims to analyze the sensitivity of the internet pricing scheme model using the modified Cloud Radio Access Network (C-RAN) model with the addition of the fair network traffic management variable and combined with the Constant Elasticity of Substitution (CES) utility function. C-RAN is a centralized radio access network to process signals and send them to the core network where the equipment used is connected to cellular antennas. The improved C-RAN model is modified into 2 cases, each of which has 3 pricing schemes, namely flat-fee, usage-based and two-part tariff, with the provision of initial usage and a predetermined amount of bandwidth consumption. This research data are secondary data obtained from the local server of local server traffic data in Palembang. Based on the analysis that has been done, the results of this study indicate that the improved C-RAN model can be utilized by Internet Service Providers (ISPs) to increase profits. The results of the sensitivity analysis for the improved model show that, if the variable is infinity, the increase and decrease are not fixed, whereas if the variable is 0.000 then the increase and decrease are fixed.
\end{abstract}

Keywords

C-RAN, Dynamic Spectrum, Traffic Management, Sensitivity Analysis, Constant of Elasticity Substitution

Received: 27 October 2021, Accepted: 08 January 2022

https://doi.org/10.26554/sti.2022.7.1.49-57

\section{INTRODUCTION}

Currently, the development of communication technology is very rapid, especially on the internet. The internet is a communication network that has the function to connect one electronic media to another quickly and precisely. All people use the internet to meet information needs in various fields such as the need for information service, the good bandwidth, and so on. Therefore, with the increasing need for the internet, the quality of service must also be improved.

As a service provider, the internet has an ISP (Internet Service Provider) (Nurajizah et al., 2020). ISP serves to distribute network connections so that users can access all the information on the internet. The internet must have the right cost planning to provide better QoS (Quality of Service) (Indrawati et al., 2020; Puspita et al., 2015; Puspita et al., 2020), so that it benefits ISPs and internet users. QoS is defined as user satisfaction, thus internet access is not only influenced by network performance but is also influenced by service performance from service providers. Besides that, the enhancement on how to promote networks of ISP to set up the best service to persuade customers joining some programs through bundling strategy and customers' selection (Indrawati et al., 2021) is also be initiated.

Based on previous research Puspita et al. (2021) which discusses internet pricing based on the Cloud Radio Access Network (C-RAN) (Indrawati et al., 2017) model combined with fair network management (Iosifidis et al., 2017; Li et al., 2016; Menth and Zeitler, 2016; Montoya et al., 2018) variables to improve services or $\mathrm{QoS}$ which focuses on internet pricing schemes (Wu et al., 2016). Dynamic Spectrum Management (DSM) Tsiaflakis et al. (2012) refers to a set of techniques to reduce the effect of crosstalk leading to the best performance. That environment involving pricing scheme will be discussed in detail along with traffic management (Sasidhar et al., 2016) of DSL-LTE (Digital Subscriber Line-Long Term Evolution) (Belghith et al., 2014; Li et al., 2016) network as an optimization problem.

In maximizing user satisfaction, 3 pricing schemes can be determined, namely flat-fee, usage-based, and two-part tariff. Flat-fee is internet pricing that has been determined every month (Indrawati et al., 2021; Wu and Banker, 2010), usage-based is internet pricing where the payment is how much 
internet access is used (Puspita et al., 2021), two-part tariff is internet pricing where the price and internet access are limited according to the user's wishes (Sitepu et al., 2019).

The C-RAN network scheme is in a critical state to be analyzed by combining the fair network model and adding the Constant Elasticity Of Substitution (CES) (Bhattacharya , 2017; Cheng, 2019; Quaas et al., 2020) utility function into the model due to its importance in recent network technology. The advantage of using the CES function is due to its capability in having a elasticity of substitution variable between inputs (Romdhoni et al., 2015). Then, the discussion of an improved model of internet pricing schemes on bandwidth consumption efficiency using the model proposed by Peng et al. (2014) is necessary to be explored. The proposed C-RAN model is then combined by adding the fair network variable.

Besides that, it is necessary to also conduct the sensitivity analysis Andersson and Rawlings (2018); Stechlinski et al. (2018); Stechlinski et al. (2019) in evaluating how much changes for the coefficients of the objective function can be allowed to maintain the optimality using LINGO 13.0 (Cunningham and Schrage, 2004).

Therefore, detailed research is needed that considers the C-RAN network in managing network traffic by utilizing the fair network combined with a pricing scheme and its sensitivity to be measured to seek optimal value ranges. This is very important for both sides (ISP and users) to gain their goals in applying the network since this model will provide benefits to maximize profits for ISPs and users to maximize its satisfaction in using the networks.

\section{EXPERIMENTAL SECTION}

\subsection{Data}

The data used are basically to validate the model that has been designed. These are secondary data obtained from a local server in Palembang. This study includes traffic data obtained from April 19, 2021 - May 18, 2021. The data are divided into 2 categories, namely peak hours and non-peak hours to show the highest level of consumption in using the network during peak and off-peak hours.

\subsection{Methods}

This research were conducted in some stages which involve some preparations such as describing the secondary data taken from one of the local server in Palembang, then the series of works in modeling the model as mixed integer nonlinear programming, computational works, and lastly the analysis which can be summarized into the steps as follows.

1. Describe the data that were carried out on one of the local server. The data are in the form of secondary data consisting of inbound and outbound traffic data.

2. Group data consisting of inbound and outbound into two categories, namely data during peak hours $(07.00-$ 17.00 Western part of Indonesian Time) and during offpeak hours (19.00 - 05.00 Western part of Indonesian Time)
3. Process data that has been grouped into 24 data where the data are $100 \mathrm{kbps}$.

4. Define the parameters and decision variables used in the C-RAN model, fair network and CES utility functions for bandwidth consumption on the network which is divided into 2 cases:

i. $B$ as constant and $D^{M}$ as variable

ii. $B$ and $D^{M}$ as constants

5. Develop an improved C-RAN model (C-RAN, fair network and utility functions (CES) for homogeneous consumers.

6. Formulate an improved C-RAN model using local server traffic data for bandwidth consumption.

7. Complete the solution of the improved C-RAN model from step 4 and step 5 using the LINGO 13.0 application software.

8. Validate as sensitivity analysis problem using LINGO solver.

9. Analyze the results obtained.

\section{RESULTS AND DISCUSSION}

\subsection{Original Model}

The original C-RAN model was modified by adding a fair network traffic management variable and then combined with CES, an improved C-RAN, fair network, and utility function of CES was formed initially by utilizing Peng et al. (2014) and modification is as follows.

$$
\operatorname{Max} \frac{\sum_{n=1}^{N+M} \sum_{k=1}^{K} a_{n, k} B \log _{2}\left(1+\sigma_{n, k} d_{n, k}\right)+\left(\sum_{i=1}^{I} v_{i}\right)^{2}}{\mu_{\mathrm{eff}} \sum_{n=1}^{N+M} \sum_{k=1}^{K} a_{n, k} d_{n, k}+D \frac{C}{R}+D_{b h}+I \sum_{i=1}^{I} v_{i}^{2}}
$$

subject to

a.

$$
\sum_{n=1}^{N+M} \sum_{k=1}^{K} a_{n, k}=1, a_{n, k} \epsilon\{0,1\}
$$

b.

$$
\sum_{n=1}^{N} C_{n, k} \geq \eta_{R}, k \epsilon \Omega_{1}
$$

c.

$$
\sum_{n=N+1}^{N+M} C_{n, k} \geq \eta_{E R}, k \epsilon \Omega_{2}
$$

d.

$$
\sum_{n=N}^{N+M} a_{n, k} d_{n, k} g_{k}^{R 2 M} t_{k}^{R 2 M} \leq \delta_{0}, k \in \Omega_{I I}
$$

e.

$$
\sum_{n=1}^{N+M} \sum_{k=1}^{K} a_{n, k} d_{n, k} \leq Q_{\max }^{R}, d_{n, k} \geq 0
$$


f.

$$
v_{i} \geq C_{i}^{\min }
$$

g.

$$
X \leq \bar{X} Z
$$

h.

$$
Y \leq \bar{Y} Z
$$

i.

$$
\ln X^{a}+\ln Y^{b}-P_{p} X-P_{n} Y-P Z \geq 0, Z=0 \text { or } 1
$$

where

j.

$$
C_{n, k}=a_{n, k} \bar{B} \log _{2}\left(1+\sigma_{n, k} d_{n, k}\right)
$$

k.

$$
\sigma_{n, k}=\left\{\begin{aligned}
\frac{g_{n}^{R} t_{n, k}^{R}}{\bar{B} N_{0}}, & k \in \Omega_{1} \\
\frac{g_{n}^{R} t_{n, k}^{R}}{H^{M} g_{N}^{R} t_{n, k}^{R}+\bar{B} N_{0}}, & k \in \Omega_{1}
\end{aligned}\right.
$$

The descriptions of the model (1)-(1k) are following.

1. Objective function (1) aims to manage the allocation of usage bandwidth with limits of N, M and K.

2. Eq. (1a) and Eq. (1f) show the limitation of RB allocation that each RB cannot be allocated more than one RUE at the same time.

3. Eq. (1b) and Eq.(1c) are set up according to high and low-level constrained QoS requirements which define minimum data rates $\beta_{R}$ and $\beta_{E R}$.

4. Eq. (1d) limits the transfer bandwidth from the RUE $n$ to the $\mathrm{RB}$ to $k$ suppress interference from the RRH to the user reusing the $\mathrm{RB}$ with $k \in \omega_{I I}$.

5. Eq. (1e) $Q_{\max }^{R}$ shows the maximum transmit power of RRH.

6. Eq. (1f) indicates that the total bandwidth received by user $i$ must be greater than the throughput minimum required of user $i$, depending on the application.

7. Eq. ( $1 \mathrm{~g})$ is determined by consumers $i$, if consumers choose not to join the program, $Z_{i}$ is 0 , so Eq. ( $1 \mathrm{~g}$ ) and (1h) for consumption levels during peak and off-peak $\left(\mathrm{X}_{i}\right.$ dan $\mathrm{Y}_{i}$ ) hours are 0 .

8. If $Z_{i}=0$ then in Eq. ( $\left.1 i\right)$, the total utility value and the costs incurred by consumers are 0 . But if consumer i chooses to join then he must decide the optimal level of consumption in peak and off-peak hours (cannot be exceed the maximum consumption limit of $\bar{X}_{i}$ and $\bar{Y}_{i}$ ).

Table 1 and Table 2 depict the parameters and variables designed for the improved model for each case.

Table 3 and Table 4 display the parameter value for each case and for homogeneous consumer. $a$ and $b$ are constant, the value $a$ and $b$ are determined provided that $a$ and $b$ are integer positive with $a>b$. with

$a$ : Service constant in peak hour, $b$ : Service constant in non-peak hours

Then, the improved cases are divided as follows based on three pricing schemes and based on $\bar{B}$ as constant or variable and $\mathrm{D}^{M}$ as a constant or Variable.

Case $1: \bar{B}$ as constant and $\mathrm{D}^{M}$ as variable

The objective function as follows:

$$
\begin{aligned}
& \operatorname{Max} \frac{\sum_{n=1}^{3+3} \sum_{k=1}^{2} a_{n, k} \bar{B} \log _{2}\left(1+\sigma_{n, k} d_{n, k}\right)+\left(\sum_{i=1}^{3} v_{i}\right)^{2}}{\mu_{\text {eff }} \sum_{n=1}^{3+3} \sum_{k=1}^{2} a_{n, k} d_{n, k}+D_{c}^{R}+D_{b h}+I \sum_{i=1}^{3} v_{i}^{2}}+\ln X^{a}-\ln Y^{b}-P_{p} X-P_{n} Y-P Z \\
& =\left[\left(5000 \log _{2}\left(1+\sigma_{11} d_{11}\right) a_{11}+5000 \log _{2}\left(1+\sigma_{12} d_{12}\right) a_{12}\right.\right. \\
& +5000 \log _{2}\left(1+\sigma_{21} d_{21}\right) a_{21}+5000 \log _{2}\left(1+\sigma_{22} d_{22}\right) a_{22} \\
& +5000 \log _{2}\left(1+\sigma_{31} d_{31}\right) a_{31}+5000 \log _{2}\left(1+\sigma_{32} d_{32}\right) a_{32} \\
& +5000 \log _{2}\left(1+\sigma_{41} d_{41}\right) a_{41}+5000 \log _{2}\left(1+\sigma_{42} d_{42}\right) a_{42} \\
& +5000 \log _{2}\left(1+\sigma_{41} d_{41}\right) a_{41}+5000 \log _{2}\left(1+\sigma_{42} d_{42}\right) a_{42} \\
& +5000 \log _{2}\left(1+\sigma_{51} d_{51}\right) a_{51}+5000 \log _{2}\left(1+\sigma_{52} d_{52}\right) a_{52} \\
& +5000 \log _{2}\left(1+\sigma_{61} d_{61}\right) a_{61}+5000 \log _{2}\left(1+\sigma_{62} d_{62}\right) a_{62}+\left(v_{1}+v_{2}+v_{3}\right)^{2} / \\
& 500\left(\left(a_{11} d_{11}+4500+4000\right)+\left(a_{12} d_{12}+4500+4000\right)\right. \\
& +\left(a_{21} d_{21}+4500+4000\right)+\left(a_{22} d_{22}+4500+4000\right) \\
& +\left(a_{31} d_{31}+4500+4000\right)+\left(a_{32} d_{32}+4500+4000\right) \\
& +\left(a_{41} d_{41}+4500+4000\right)+\left(a_{42} d_{42}+4500+4000\right) \\
& +\left(a_{51} d_{51}+4500+4000\right)+\left(a_{52} d_{52}+4500+4000\right) \\
& \left.+\left(a_{61} d_{61}+4500+4000\right)+\left(a_{62} d_{62}+4500+4000\right)\right) \\
& \left.+3\left(v_{1}^{2}+v_{2}^{2}+v_{3}^{2}\right)\right]-\ln X^{a}-\ln Y^{b}-P_{p} X-P_{n} Y-P Z
\end{aligned}
$$

Based on Eq.(1a):

$a_{11}+a_{12}+a_{21}+a_{22}+a_{31}+a_{32}+a_{41}+a_{42}+a_{51}+a_{52}+a_{61}+a_{62}=1$

Based on (1b):

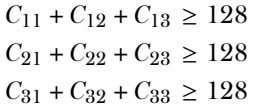

$$
\begin{gathered}
a_{11} 5000 \log _{2}\left(1+\frac{(6081.85)(245.4)}{5000 N_{0}} d_{11}\right)+ \\
a_{12} 5000 \log _{2}\left(1+\frac{(6081.85)(353.56)}{5000 N_{0}} d_{12}\right)+
\end{gathered}
$$$$
a_{13} 5000 \log _{2}\left(1+\frac{(6081.85)(670.127)}{5000 N_{0}} d_{13}\right) \geq 128
$$

$$
a_{21} 5000 \log _{2}\left(1+\frac{(549.49)(377.24)}{5000 N_{0}} d_{21}\right)+
$$$$
a_{22} 5000 \log _{2}\left(1+\frac{(549.49)(257.04)}{5000 N_{0}} d_{22}\right)+
$$

$$
a_{31} 5000 \log _{2}\left(1+\frac{(17.346)(249.38)}{5000 N_{0}} d_{31}\right)+
$$$$
a_{32} 5000 \log _{2}\left(1+\frac{(17.346)(253.95)}{5000 N_{0}} d_{32}\right)+
$$$$
a_{33} 5000 \log _{2}\left(1+\frac{(17.346)(392.5)}{5000 N_{0}} d_{33}\right) \geq 128
$$ 
Table 1. Parameters for Each Case

\begin{tabular}{|c|c|}
\hline & Case 1 : as constants and as variable \\
\hline $\bar{B}$ & : ISP-defined bandwidth setting \\
\hline$\mu_{\text {eff }}$ & : Pricing for bandwidth (IDR) \\
\hline $\mathrm{D}_{C}^{R}$ & : During peak hours, bandwidth usage is limited \\
\hline $\mathrm{D}_{b h}^{c}$ & : Limits on Off-peak bandwidth utilization \\
\hline$\eta_{R}$ & : Maximum level of QoS \\
\hline$\eta_{E R}$ & : Minimum level of QoS \\
\hline$\delta_{0}$ & : The user's maximum bandwidth usage limit \\
\hline $\mathrm{D}_{\max }^{R}$ & : Transfer bandwidth at its maximum \\
\hline $\begin{array}{l}\mathrm{g}_{n}^{R} \\
\mathrm{t}_{n, k}^{R}\end{array}$ & $\begin{array}{l}\text { : Amount of maximum bandwidth usage and minimum bandwidth usage } \\
\text { : A total of daily bandwidth usage (Kbps) }\end{array}$ \\
\hline $\begin{array}{c}c_{i, k} \text { min } \\
c_{i}\end{array}$ & : Minimum throughput received by user i \\
\hline $\mathrm{P}^{2}$ & : Costs that consumer will incur to join the service \\
\hline $\mathrm{P}_{p}$ & : During peak hours, the service provider sets the unit price. \\
\hline & : During non-peak hours, the service provider sets the unit price. \\
\hline $\mathrm{U}_{i\left(x_{i}, y_{i}\right)}$ & : Utility function belongs to customer $i$ in rates of peak and off-peak consumption \\
\hline $\bar{B}$ & $\begin{array}{l}\text { Case } 2: \text { and as constants } \\
\text { : ISP-defined bandwidth setting }\end{array}$ \\
\hline$\mu_{\text {eff }}$ & : Pricing for bandwidth (IDR) \\
\hline $\mathrm{D}_{C}^{R}$ & : During peak hours, bandwidth usage is limited \\
\hline $\mathrm{D}_{b h}$ & : Limits on Off-peak bandwidth utilization \\
\hline$\eta_{R}$ & : Maximum level of QoS \\
\hline$\eta_{E R}$ & : Minimum level of QoS \\
\hline$\delta_{0}$ & : Bandwidth Usage highest limit consumed by user \\
\hline $\mathrm{D}_{\max }^{R}$ & : The highest transfer bandwidth \\
\hline $\begin{array}{l}\mathrm{g}_{n}^{R} \\
\mathrm{t}_{n, k}^{R}\end{array}$ & $\begin{array}{l}\text { : Amount of highest and lowest consumption of bandwidth } \\
\text { : Whole daily bandwidth usage (Kbps) }\end{array}$ \\
\hline $\begin{array}{l}n, k \\
\mathrm{D}^{M}\end{array}$ & : Initial bandwidth consumption \\
\hline $\mathrm{c}_{i}^{\min }$ & : Minimum throughput accepted by user $\mathrm{i}$ \\
\hline $\mathrm{P}^{2}$ & : Costs for customer in joining the service \\
\hline $\mathrm{P}_{p}$ & : During peak hours, the service provider sets the unit price. \\
\hline & : During non-peak hours, the service provider sets the unit price. \\
\hline $\mathrm{U}_{i\left(x_{i}, y_{i}\right)}$ & : Consumer utility function i for peak and off-peak consumption levels \\
\hline
\end{tabular}

Based on Eq.(1c):

$C_{44}+C_{45}+C_{46} \geq 64 C_{54}+C_{55}+C_{56} \geq 64 C_{64}+C_{65}+C_{66} \geq 64$

Based on Eq.(1c), (1j) and (1k):

$$
\begin{array}{r}
a_{44} 5000 \log _{2}\left(1+\frac{(264.44)(170.99)}{\left(D^{M} g_{4}^{M} t_{44}^{M}+5000 N_{0}\right)} d_{44}\right)+ \\
a_{45} 5000 \log _{2}\left(1+\frac{(264.44)(342.45)}{\left(D^{M} g_{4}^{M} t_{45}^{M}+5000 N_{0}\right)} d_{45}\right)+ \\
a_{46} 5000 \log _{2}\left(1+\frac{(264.44)(125.44)}{\left(D^{M} g_{4}^{M} t_{46}^{M}+5000 N_{0}\right)} d_{46}\right) \geq 64 \\
a_{54} 5000 \log _{2}\left(1+\frac{(91.8182)(335.49)}{\left(D^{M} g_{5}^{M} t_{54}^{M}+5000 N_{0}\right)} d_{54}\right)+ \\
a_{55} 5000 \log _{2}\left(1+\frac{(91.8182)(6107.55)}{\left(D^{M} g_{5}^{M} t_{55}^{M}+5000 N_{0}\right)} d_{55}\right)+ \\
a_{56} 5000 \log _{2}\left(1+\frac{(91.8182)(144.5571)}{\left(D^{M} g_{5}^{M} t_{56}^{M}+5000 N_{0}\right)} d_{56}\right) \geq 64 \\
a_{64} 5000 \log _{2}\left(1+\frac{(14.3824)(119.7607)}{\left(D^{M} g_{6}^{M} t_{64}^{M}+5000 N_{0}\right)} d_{64}\right)+ \\
a_{65} 5000 \log _{2}\left(1+\frac{(14.3824)(273.38)}{\left(D^{M} g_{6}^{M} t_{65}^{M}+5000 N_{0}\right)} d_{65}\right)+ \\
a_{66} 5000 \log _{2}\left(1+\frac{(14.3824)(365.36)}{\left(D^{M} g_{6}^{M} t_{66}^{M}+5000 N_{0}\right)} d_{66}\right) \geq 64
\end{array}
$$

Based on Eq.(1d): $a_{31} d_{31} g_{1}^{R 2 M} t_{1}^{R 2 M}+a_{41} d_{41} g_{1}^{R 2 M} t_{1}^{R 2 M}+a_{51} d_{51} g_{1}^{R 2 M} t_{1}^{R 2 M}+a_{61} d_{61} g_{1}^{R 2 M} t_{1}^{R 2 M} \leq 4500$
$a_{32} d_{32} g_{1}^{R 2 M} t_{1}^{R 2 M}+a_{42} d_{42} g_{1}^{R 2 M} t_{1}^{R 2 M}+a_{52} d_{52} g_{1}^{R 2 M} t_{1}^{R 2 M}+a_{62} d_{62} g_{1}^{R 2 M} t_{1}^{R 2 M} \leq 4500$
$a_{33} d_{33} g_{1}^{R 2 M} t_{1}^{R 2 M}+a_{43} d_{43} g_{1}^{R 2 M} t_{1}^{R 2 M}+a_{53} d_{53} g_{1}^{R 2 M} t_{1}^{R 2 M}+a_{63} d_{63} g_{1}^{R 2 M} t_{1}^{R 2 M} \leq 4500$
$a_{34} d_{34} g_{1}^{R 2 M} t_{1}^{R 2 M}+a_{44} d_{44} g_{1}^{R 2 M} t_{1}^{R 2 M}+a_{54} d_{54} g_{1}^{R 2 M} t_{1}^{R 2 M}+a_{64} d_{64} g_{1}^{R 2 M} t_{1}^{R 2 M} \leq 4500$
$a_{35} d_{35} g_{1}^{R 2 M} t_{1}^{R 2 M}+a_{45} d_{45} g_{1}^{R 2 M} t_{1}^{R 2 M}+a_{55} d_{55} g_{1}^{R 2 M} t_{1}^{R 2 M}+a_{65} d_{65} g_{1}^{R 2 M} t_{1}^{R 2 M} \leq 4500$
$a_{36} d_{36} g_{1}^{R 2 M} t_{1}^{R 2 M}+a_{46} d_{46} g_{1}^{R 2 M} t_{1}^{R 2 M}+a_{56} d_{56} g_{1}^{R 2 M} t_{1}^{R 2 M}+a_{66} d_{66} g_{1}^{R 2 M} t_{1}^{R 2 M} \leq 4500$

Based on Eq.(1e):

$$
\begin{array}{r}
a_{11} d_{11}+a_{12} d_{12}+a_{21} d_{21}+a_{22} d_{22}+a_{31} d_{31}+a_{32} d_{32}+a_{41} \\
d_{41}+a_{42} d_{42}+a_{51} d_{51}+a_{52} d_{52}+a_{61} d_{61}+a_{62} d_{62} \leq 500
\end{array}
$$

Based on Eq.(1f) :

$$
\begin{aligned}
& v_{1} \geq 119.7 \\
& v_{2} \geq 119.7 \\
& v_{3} \geq 119.7
\end{aligned}
$$

Based on Eq.(1g):

$$
X \leq 6081.85 Z
$$


Table 2. Variable Decision for Each Case

\begin{tabular}{|c|c|}
\hline \multicolumn{2}{|r|}{ Case 1 : as constants and as variable } \\
\hline $\mathrm{a}_{n, k}$ & : Indicator of RB allocation which is having 0 or 1 \\
\hline $\mathrm{d}_{n, k}$ & : Bandwidth transfer from RB to RUE \\
\hline $\mathrm{g}_{k}^{R 2 M}$ & : Corresponding path loss of RRH on RB \\
\hline $\mathrm{t}_{k}^{K 2 M}$ & : Corresponding channel gain of RRH on RB \\
\hline $\mathrm{D}_{M}$ & : Initial bandwidth usage \\
\hline $\mathrm{g}_{n}^{M}$ & : Loss of path from RB to RUE \\
\hline $\mathrm{t}_{n, k}^{\dot{M}}$ & : Gain of channel from RB to RUE \\
\hline $\mathrm{N}_{0}$ & : The usage of Bandwidth when it is not in hosting condition \\
\hline $\mathrm{v}_{i}$ & : Total bandwidth received by user i \\
\hline $\mathrm{X}_{i}$ & : Consumption level of consumer i in peak hour service \\
\hline $\mathrm{Y}_{i}$ & : Consumer consumption level $\mathrm{i}$ in off-peak hours \\
\hline $\bar{X}_{i}$ & : The maximum consumption rate of consumer i during peak hours \\
\hline $\bar{Y}_{i}$ & $\begin{array}{l}\text { : Maximum consumption level of consumer i during off-peak hours } \\
\text { Case } 2: \text { and as constants }\end{array}$ \\
\hline $\mathrm{a}_{n, k}$ & : RB allocation indicator of 0 or 1 \\
\hline $\mathrm{d}_{n, k}^{n, n}$ & : Bandwidth transfer from RB to RUE \\
\hline $\mathrm{g}_{k}^{R 2 M}$ & : Corresponding path loss of RRH on RB \\
\hline $\mathrm{t}_{k}^{k 2 M}$ & : Corresponding channel gain of RRH on RB \\
\hline${ }_{\mathrm{g}_{n}}^{M}$ & : Path loss value from RB to RUE \\
\hline $\mathrm{t}_{n, k}^{M}$ & : Channel gain value from RB to RUE \\
\hline $\mathrm{N}_{0}$ & : Bandwidth usage when not hosting \\
\hline $\mathrm{v}_{i}$ & : Total bandwidth received by user i \\
\hline $\mathrm{X}_{i}$ & : Consumption level of consumer i in peak hour service \\
\hline $\mathrm{Y}_{i}$ & : Consumer consumption level i in off-peak hours \\
\hline $\bar{X}_{i}$ & : he maximum consumption rate of consumer i during peak hours \\
\hline $\bar{Y}_{i}$ & : Maximum consumption level of consumer i during off-peak hours \\
\hline
\end{tabular}

Based on Eq.(1h):

$Y \leq 264.44 Z$

Based on Eq.(1i):

$$
\ln X^{a}-\ln Y^{b}-P_{p} X-P_{n} Y-P Z \geq 0
$$

Based on Eq.(1i), selected $Z=1$ value which means customers choose to join the offered program, with the following pricing scheme

If pricing scheme of flat-fee then add the constraints as following.

$$
\begin{aligned}
P_{p} & =0 \\
P_{n} & =0 \\
P & >0
\end{aligned}
$$

If pricing scheme of usage-based add constraints as following.

$$
\begin{array}{r}
P_{p}>0 \\
P_{n}>0 \\
P=0
\end{array}
$$

If pricing scheme of two-part tariff add constraints as following.

$$
\begin{aligned}
P_{p} & >0 \\
P_{n} & >0 \\
P & >0
\end{aligned}
$$

Case $2 \bar{B}$ and $\mathrm{D}^{M}$ as constants

The objective function as follows:

$$
\begin{aligned}
& \operatorname{Max} \frac{\sum_{n=1}^{3+3} \sum_{k=1}^{2} a_{n, k} B \log _{2}(1+}{\left.\sigma_{n, k} d_{n, k}\right)+\left(\sum_{i=1}^{3} v_{i}\right)^{2}}+\ln X^{a}-\ln Y^{b}-P_{p} X-P_{n} Y-P Z \\
& \mu_{\mathrm{eff}} \sum_{n=1}^{3+3} \sum_{k=1}^{2} a_{n, k} d_{n, k}+D_{b h}+^{I} \sum_{i=1}^{3} v_{i}^{2} \\
&=\left(5000 \log _{2}\left(1+\sigma_{11} d_{11}\right) a_{11}+5000 \log _{2}\left(1+\sigma_{12} d_{12}\right) a_{12}\right. \\
&+5000 \log _{2}\left(1+\sigma_{21} d_{21}\right) a_{21}+5000 \log _{2}\left(1+\sigma_{22} d_{22}\right) a_{22} \\
&+ 5000 \log _{2}\left(1+\sigma_{31} d_{31}\right) a_{31}+5000 \log _{2}\left(1+\sigma_{32} d_{32}\right) a_{32} \\
&+5000 \log _{2}\left(1+\sigma_{41} d_{41}\right) a_{41}+5000 \log _{2}\left(1+\sigma_{42} d_{42}\right) a_{42} \\
&+ 5000 \log _{2}\left(1+\sigma_{51} d_{51}\right) a_{51}+5000 \log _{2}\left(1+\sigma_{52} d_{52}\right) a_{52} \\
&+5000 \log _{2}\left(1+\sigma_{61}\right.\left.d_{61}\right) a_{61}+5000 \log _{2}\left(1+\sigma_{62} d_{62}\right) a_{62}+\left(v_{1}+v_{2}+v_{3}\right)^{2} / \\
& 500\left(\left(a_{11} d_{11}+4500+4000\right)+\left(a_{12} d_{12}+4500+4000\right)\right. \\
&+\left(a_{21} d_{21}+4500+4000\right)+\left(a_{22} d_{22}+4500+4000\right) \\
&+\left(a_{31} d_{31}+4500+4000\right)+\left(a_{32} d_{32}+4500+4000\right) \\
&+\left(a_{41} d_{41}+4500+4000\right)+\left(a_{42} d_{42}+4500+4000\right) \\
&+\left(a_{51} d_{51}+4500+4000\right)+\left(a_{52} d_{52}+4500+4000\right) \\
&+\left(a_{61} d_{61}+4500+4000\right)+\left(a_{62} d_{62}+4500+4000\right) \\
&\left.+3\left(v_{12}+v_{22}+v_{32}\right)\right]-\ln X^{a}-\ln Y^{b}-P_{p} X-P_{n} Y-P Z
\end{aligned}
$$

Based on Eq. (1a):

$a_{11}+a_{12}+a_{21}+a_{22}+a_{31}+a_{32}+a_{41}+a_{42}+a_{51}+a_{52}+a_{61}+a_{62}=1$

Based on Eq. (1b):

$$
\begin{aligned}
& C_{11}+C_{12}+C_{13} \geq 128 \\
& C_{21}+C_{22}+C_{13} \geq 128 \\
& C_{31}+C_{32}+C_{33} \geq 128
\end{aligned}
$$


Table 3. Parameter Value for Each Case

\begin{tabular}{|c|c|}
\hline Parameter & Value \\
\hline $\mathrm{g}_{1}^{R}=\bar{X}_{1}$ & $6,081.85$ \\
\hline$g_{2}^{k}=\bar{X}_{2}$ & 549.49 \\
\hline $\mathrm{g}_{3}^{R}=\bar{X}_{3}$ & 17.346 \\
\hline $\mathrm{g}_{4}^{\mathcal{R}}=\bar{X}_{4}$ & 264.44 \\
\hline $\mathrm{g}_{5}^{R}=\bar{X}_{5}$ & 91.8182 \\
\hline $\mathrm{g}_{6}^{R}=\bar{X}_{6}$ & 14.3824 \\
\hline $\mathrm{t}_{11}^{R}$ & 245.4 \\
\hline $\mathrm{t}_{12}^{\mathrm{R}}$ & 353.56 \\
\hline$t_{21}^{k^{2}}$ & 377.24 \\
\hline$t_{22}^{R}$ & 257.04 \\
\hline$t_{31}^{k}$ & 249.38 \\
\hline $\mathrm{t}_{32}^{K^{2}}$ & 253.95 \\
\hline $\mathrm{t}_{41}^{R}$ & 527.342 \\
\hline $\mathrm{t}_{42}^{K^{2}}$ & 381.38 \\
\hline$t_{51}^{k^{2}}$ & 465.64 \\
\hline $\mathrm{t}_{59}^{K^{1}}$ & 586.69 \\
\hline$t_{6}^{K^{2}}$ & 433.28 \\
\hline$t_{62}^{K^{2}}$ & 147.56 \\
\hline$t_{13}^{K^{2}}$ & 670.127 \\
\hline$t_{93}^{k^{\circ}}$ & 167.05 \\
\hline$t_{33}^{K}$ & 392.5 \\
\hline$K^{K}$ & 170.99 \\
\hline${ }_{R}^{R} R$ & 342.45 \\
\hline$t_{46}^{R}$ & 125.44 \\
\hline $\mathrm{t}_{54}^{\mathrm{R}}$ & 335.49 \\
\hline $\mathrm{t}_{55}^{\mathrm{R}^{4}}$ & $6,107.55$ \\
\hline $\mathrm{t}_{56}^{\mathrm{R}}$ & 144.5571 \\
\hline $\mathrm{t}_{64}^{\mathrm{R}}$ & 119.7607 \\
\hline $\mathrm{t}_{65}^{R}$ & 273.38 \\
\hline $\mathrm{t}_{66}^{k^{2}}$ & 365.36 \\
\hline
\end{tabular}

Based on (1b), (1j), and (1k)

$$
\begin{array}{r}
a_{11} 5000 \log _{2}\left(1+\frac{(6081.85)(245.4)}{5000 N_{0}} d_{11}\right)+ \\
a_{12} 5000 \log _{2}\left(1+\frac{(6081.85)(353.56)}{5000 N_{0}} d_{12}\right)+ \\
a_{13} 5000 \log _{2}\left(1+\frac{(6081.85)(670.127)}{5000 N_{0}} d_{13}\right) \geq 128 \\
a_{21} 5000 \log _{2}\left(1+\frac{(549.49)(377.24)}{5000 N_{0}} d_{21}\right)+ \\
a_{22} 5000 \log _{2}\left(1+\frac{(549.49)(257.04)}{5000 N_{0}} d_{22}\right)+ \\
a_{23} 5000 \log _{2}\left(1+\frac{(549.49)(167.05)}{5000 N_{0}} d_{23}\right) \geq 128 \\
a_{31} 5000 \log _{2}\left(1+\frac{(17.346)(249.38)}{5000 N_{0}} d_{31}\right)+ \\
a_{32} 5000 \log _{2}\left(1+\frac{(17.346)(253.95)}{5000 N_{0}} d_{32}\right)+ \\
a_{33} 5000 \log _{2}\left(1+\frac{(17.346)(392.5)}{5000 N_{0}} p_{33}\right) \geq 128
\end{array}
$$

Table 4. Parameter Value of Homogeneous Consumers

\begin{tabular}{cccc}
\hline \multirow{2}{*}{ Parameter } & \multicolumn{3}{c}{ Pricing Scheme } \\
& Flat-Fee & Usage-Based & Two-Part Tariff \\
\hline$\alpha$ & 4 & 4 & 4 \\
$\beta$ & 3 & 3 & 4 \\
$\bar{X}$ & $6,081.85$ & $6,081.85$ & $6,081.85$ \\
$\bar{Y}$ & 264.44 & 264.44 & 264.44 \\
\hline
\end{tabular}

Based on (1c) :

$$
\begin{aligned}
& C_{44}+C_{45}+C_{46} \geq 64 \\
& C_{54}+C_{55}+C_{56} \geq 64 \\
& C_{64}+C_{65}+C_{66} \geq 64
\end{aligned}
$$

Based on (1c), (1j) and (1k):

$$
\begin{gathered}
a_{44} 5000 \log _{2}\left(1+\frac{(264.44)(170.99)}{\left(150 g_{4}^{M} t_{44}^{M}+5000 N_{0}\right)} d_{44}\right)+ \\
a_{45} 5000 \log _{2}\left(1+\frac{(264.44)(342.45)}{\left(150 g_{4}^{M} t_{45}^{M}+5000 N_{0}\right)} d_{45}\right)+ \\
a_{46} 5000 \log _{2}\left(1+\frac{(264.44)(125.44)}{\left(150 g_{4}^{M} t_{46}^{M}+5000 N_{0}\right)} d_{46}\right) \geq 64 \\
a_{54} 5000 \log _{2}\left(1+\frac{(91.8182)(335.49)}{\left(150 g_{5}^{M} t_{54}^{M}+5000 N_{0}\right)} d_{54}\right)+ \\
a_{55} 5000 \log _{2}\left(1+\frac{(91.8182)(6107.55)}{\left(150 g_{5}^{M} t_{55}^{M}+5000 N_{0}\right)} d_{55}\right)+ \\
a_{56} 5000 \log _{2}\left(1+\frac{(91.8182)(144.5571)}{\left(150 g_{5}^{M} t_{56}^{M}+5000 N_{0}\right)} d_{56}\right) \geq 64 \\
a_{64} 5000 \log _{2}\left(1+\frac{(14.3824)(119.7607)}{\left(150 g_{6}^{M} t_{64}^{M}+5000 N_{0}\right)} d_{64}\right)+ \\
a_{65} 5000 \log _{2}\left(1+\frac{(14.3824)(273.38)}{\left(150 g_{6}^{M} t_{65}^{M}+5000 N_{0}\right)} d_{65}\right)+ \\
a_{66} 5000 \log _{2}\left(1+\frac{(14.3824)(365.36)}{\left(150 g_{6}^{M} t_{66}^{M}+5000 N_{0}\right)} d_{66}\right) \geq 64
\end{gathered}
$$

Based on Eq.(1d):

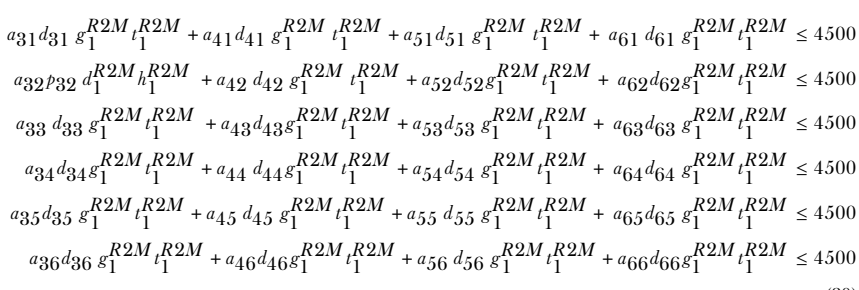

Based on Eq. (1e):

$a_{11} d_{11}+a_{12} d_{12}+a_{21} d_{21}+a_{22} d_{22}+a_{31} d_{31}+a_{32} d_{32}+a_{41}$ $d_{41}+a_{42} d_{42}+a_{51} d_{51}+a_{52} d_{52}+a_{61} d_{61}+a_{62} d_{62} \leq 500$ 
Table 5. Optimal Solution Improved C-RAN and Fair Network Models

\begin{tabular}{|c|c|c|c|c|c|c|}
\hline \multirow[t]{2}{*}{ Status of Solver } & \multicolumn{2}{|c|}{ Flat-Fee } & \multicolumn{2}{|c|}{$\begin{array}{c}\text { Scheme } \\
\text { Usage-Based }\end{array}$} & \multicolumn{2}{|c|}{ Two-Part Tariff } \\
\hline & Case 1 & Case 2 & Case 1 & Case 2 & Case 1 & Case 2 \\
\hline Model Class & NLP & NLP & NLP & NLP & NLP & NLP \\
\hline State & Local Optimal & Local Optimal & Local Optimal & Local Optimal & Local Optimal & Local Optimal \\
\hline Objective & 2 & 2 & 2 & 2 & 2 & 2 \\
\hline Infeasibility & 0 & $0.77925 \times 10^{-7}$ & 0 & 0 & 0 & $1.35956 \times 10^{-9}$ \\
\hline Iterations & 97 & 114 & 106 & 98 & 92 & 126 \\
\hline \multicolumn{7}{|c|}{ Extended Solver Status } \\
\hline Type of Solver & \multicolumn{6}{|c|}{ Branch and Bound } \\
\hline Best Objective & - & - & - & - & - & - \\
\hline Steps & - & - & - & - & - & - \\
\hline Update Interval & 2 & 2 & 2 & 2 & 2 & 2 \\
\hline GMU (K) & 69 & 69 & 69 & 69 & 69 & 69 \\
\hline ER (Sec) & 1 & 1 & 1 & 1 & 0 & 1 \\
\hline
\end{tabular}

Based on Eq.(1f):

$$
\begin{aligned}
& v_{1} \geq 119.7 \\
& v_{2} \geq 119.7 \\
& v_{3} \geq 119.7
\end{aligned}
$$

Based on Eq.( $1 g)$ :

$$
X \leq 6081.85 Z
$$

Based on Eq.(1h)

$$
Y \leq 264.44 Z
$$

Based on Eq.(11)

$$
\ln X^{a}-\ln Y^{b}-P_{p} X-P_{n} Y-P Z \geq 0
$$

Based on Eq.(1i) selected $Z=1$ value which means costumers choose to join the offered program, with the following pricing scheme:

If pricing scheme of flat-fee add the constraints as follows.

$$
\begin{aligned}
P_{p} & =0 \\
P_{n} & =0 \\
P & >0
\end{aligned}
$$

\begin{tabular}{|c|c|c|c|}
\hline Variable & $\begin{array}{c}\text { Current } \\
\text { Coefficient }\end{array}$ & $\begin{array}{l}\text { Allowable } \\
\text { Increase }\end{array}$ & $\begin{array}{l}\text { Allowable } \\
\text { Decrease }\end{array}$ \\
\hline $\mathrm{n}$ & NONLINEAR & 0.000000 & $\infty$ \\
\hline $\mathrm{a}_{12}$ & NONLINEAR & 0.000000 & $\infty$ \\
\hline$a_{21}$ & NONLINEAR & 0.000000 & $\infty$ \\
\hline$a_{22}$ & NONLINEAR & 0.000000 & $\infty$ \\
\hline$a_{32}$ & NONLINEAR & 0.000000 & $\infty$ \\
\hline$a_{41}$ & NONLINEAR & 0.000000 & $\infty$ \\
\hline $\mathrm{a}_{42}$ & NONLINEAR & 0.000000 & $\infty$ \\
\hline$a_{51}$ & NONLINEAR & 0.000000 & to \\
\hline$a_{52}$ & NONLINEAR & 0.000000 & $\infty$ \\
\hline$a_{61}$ & NONLINEAR & 0.000000 & $\infty$ \\
\hline$a_{62}$ & NONLINEAR & 0.000000 & $\infty$ \\
\hline $\mathrm{a}$ & NONLINEAR & 0.000000 & $\infty$ \\
\hline $\mathrm{X}_{A}$ & 0.000000 & $\infty$ & 0.000000 \\
\hline $\mathrm{Y}_{B}$ & 0.000000 & $\infty$ & 0.000000 \\
\hline $\mathrm{P}$ & -1.000000 & $\infty$ & -1.000000 \\
\hline a 33 & NONLINEAR & 0.000000 & $\infty$ \\
\hline$a_{44}$ & NONLINEAR & 0.000000 & $\infty$ \\
\hline $\mathrm{a}_{45}$ & NONLINEAR & 0.000000 & $\infty$ \\
\hline $\mathrm{a}_{54}$ & NONLINEAR & 0.000000 & $\infty$ \\
\hline $\mathrm{a}_{55}$ & NONLINEAR & 0.000000 & $\infty$ \\
\hline$a_{65}$ & NONLINEAR & 0.000000 & $\infty$ \\
\hline$a_{66}$ & NONLINEAR & 0.000000 & $\infty$ \\
\hline$P_{\text {MAX }}$ & 0.000000 & 0.000000 & to \\
\hline
\end{tabular}

If pricing scheme of usage-based add the constraints as follows.

$$
\begin{array}{r}
P_{p}>0 \\
P_{n}>0 \\
P=0
\end{array}
$$

If pricing scheme of two-part tariff add the constraints as follows.

$$
\begin{aligned}
P_{p} & >0 \\
P_{n} & >0 \\
P & >0
\end{aligned}
$$

Table 6. Flat-Fee Sensitivity Analysis Result for Objective Coefficient Ranges

Table 5 shows the optimal solution obtained from the solver LINGO 13.0 for the C-RAN, fair network, and CES utility 
functions models using parameter values and data traffic in each case. For this type of pricing flat-fee, the optimal solution is IDR $2 / \mathrm{Kbps}$ obtained through 97 iterations in case 1 and 114 in case 2 and Infeasibility is 0.000000 for case 1 and $2.77925 \times 10^{-7}$ for case 2 . Extended solver status shows the method used in this model is the Branch and Bound method. Steps show the number of steps for this method which does not exist for case 1 and case 2. Generated Memory Used (GMU) shows the amount of memory allocation used is $69 \mathrm{~K}$ and the length of time used to complete and generate the model is expressed in Elapsed Runtime (ER) which is equal to 1 second.

Table 7. Two-Part Tariff Sensitivity Analysis Result for Objective Coefficient Ranges

\begin{tabular}{cccc}
\hline Variabel & $\begin{array}{c}\text { Current } \\
\text { Coefficient }\end{array}$ & $\begin{array}{c}\text { Allowable } \\
\text { Increase }\end{array}$ & $\begin{array}{c}\text { Allowable } \\
\text { Decrease }\end{array}$ \\
\hline $\mathrm{n}$ & NONLINEAR & 0.000000 & $\infty$ \\
$\mathrm{a}_{12}$ & NONLINEAR & 0.000000 & $\infty$ \\
$\mathrm{a}_{21}$ & NONLINEAR & 0.000000 & $\infty$ \\
$\mathrm{a}_{22}$ & NONLINEAR & 0.000000 & $\infty$ \\
$\mathrm{a}_{32}$ & NONLINEAR & 0.000000 & $\infty$ \\
$\mathrm{a}_{41}$ & NONLINEAR & 0.000000 & $\infty$ \\
$\mathrm{a}_{42}$ & NONLINEAR & 0.000000 & $\infty$ \\
$\mathrm{a}_{51}$ & NONLINEAR & 0.000000 & $\infty$ \\
$\mathrm{a}_{52}$ & NONLINEAR & 0.000000 & $\infty$ \\
$\mathrm{a}_{61}$ & NONLINEAR & 0.000000 & $\infty$ \\
$\mathrm{a}_{62}$ & NONLINEAR & 0.000000 & $\infty$ \\
$\mathrm{a}$ & NONLINEAR & 0.000000 & $\infty$ \\
$\mathrm{P}_{X}$ & NONLINEAR & $\infty$ & 0.000000 \\
$\mathrm{X}_{A}$ & NONLINEAR & $\infty$ & $\infty$ \\
$\mathrm{P}_{Y}$ & NONLINEAR & $\infty$ & 0.000000 \\
$\mathrm{Y}_{B}$ & NONLINEAR & $\infty$ & 0.000000 \\
$\mathrm{p}$ & -1.000000 & tak hingga & -1.000000 \\
$\mathrm{a}_{33}$ & NONLINEAR & 0.000000 & $\infty$ \\
$\mathrm{a}_{44}$ & NONLINEAR & 0.000000 & $\infty$ \\
$\mathrm{a}_{45}$ & NONLINEAR & 0.000000 & $\infty$ \\
$\mathrm{a}_{54}$ & NONLINEAR & 0.000000 & $\infty$ \\
$\mathrm{a}_{55}$ & NONLINEAR & 0.000000 & $\infty$ \\
$\mathrm{a}_{54}$ & NONLINEAR & 0.000000 & $\infty$ \\
$\mathrm{a}_{66}$ & NONLINEAR & 0.000000 & $\infty$ \\
$\mathrm{P}_{\mathrm{M} A X}$ & 0.000000 & 0.000000 & $\infty$ \\
\hline & & &
\end{tabular}

This type of pricing usage-based produces an optimal solution for cases 1 and 2 of IDR 2/Kbps obtained through 106 iterations in iteration 1 and 98 in case 2. Infeasibility is 0.000000 . Extended solver status indicates the method used is Branch and Bound. Steps indicate the number of steps for this method, this does not exist. GMU is $69 \mathrm{~K}$ and ER is 1 second. The type of pricing is two-part tariff, resulting in an optimal solution for cases 1 and 2 of IDR. 2/Kbps obtained through 92 iterations for case 1 and 196 iterations for case 2 . Infeasibility is 0.000000 for case 1 and $1.35956 \times 10^{-9}$ for case 2 . Extended solver status indicates the method used is Branch and Bound. Steps indicate the number of steps for this method does not exist. GMU is $69 \mathrm{~K}$. ER is 0 seconds for case 1 and 1 second for case 2 .

Table 8. Usage-Based Sensitivity Analysis Result for Objective Coefficient Ranges

\begin{tabular}{cccc}
\hline Variable & $\begin{array}{c}\text { Current } \\
\text { Coefficient }\end{array}$ & $\begin{array}{c}\text { Allowable } \\
\text { Increase }\end{array}$ & $\begin{array}{c}\text { Allowable } \\
\text { Decrease }\end{array}$ \\
\hline $\mathrm{a}_{11}$ & NONLINEAR & 0.000000 & $\infty$ \\
$\mathrm{n}$ & NONLINEAR & 0.000000 & $\infty$ \\
$\mathrm{a}_{12}$ & NONLINEAR & 0.000000 & $\infty$ \\
$\mathrm{a}_{31}$ & NONLINEAR & 0.000000 & $\infty$ \\
$\mathrm{d}_{31}$ & NONLINEAR & $\infty$ & 0.000000 \\
$\mathrm{a}_{41}$ & NONLINEAR & 0.000000 & $\infty$ \\
$\mathrm{a}_{42}$ & NONLINEAR & 0.000000 & $\infty$ \\
$\mathrm{a}$ & NONLINEAR & 0.000000 & $\infty$ \\
$\mathrm{Y}_{B}$ & NONLINEAR & $\infty$ & 0.000000 \\
$\mathrm{a}_{33}$ & NONLINEAR & 0.000000 & $\infty$ \\
$\mathrm{a}_{44}$ & NONLINEAR & 0.000000 & $\infty$ \\
$\mathrm{a}_{45}$ & NONLINEAR & 0.000000 & $\infty$ \\
$\mathrm{a}_{54}$ & NONLINEAR & 0.000000 & $\infty$ \\
$\mathrm{a}_{55}$ & NONLINEAR & 0.000000 & $\infty$ \\
$\mathrm{a}_{66}$ & NONLINEAR & 0.000000 & $\infty$ \\
\hline
\end{tabular}

Next, the sensitivity analysis for three pricing schemes is described. The sensitivity analysis is based on LINGO 13.0 to seek for evaluation of the variable decision values. Based on Table 6 , the result of the sensitivity analysis with the flat-fee pricing scheme showed that the variable $\mathrm{n}, \mathrm{a}_{12}, \mathrm{a}_{21}, \mathrm{a}_{22}, \mathrm{a}_{32}$, $\mathrm{a}_{41}, \mathrm{a}_{42}, \mathrm{a}_{51}, \mathrm{a}_{52}, \mathrm{a}_{61}, \mathrm{a}_{62}$, has the allowable increase of 0.000 which means that the increase in the value of the variable is fixed and cannot be changed, while the allowable decrease is infinity which means that the value decreases, this variable may or may not change. In the variable $\mathrm{X}_{A}, \mathrm{Y}_{B}$, the allowable increase is infinity which means that the increase in the value of the variable can be changed or not fixed, while the allowable decrease is 0.000 which means that the decrease in the value of the variable is fixed and cannot be changed. In the variable $\mathrm{a}_{33}$, $\mathrm{a}_{44}, \mathrm{a}_{45}, \mathrm{a}_{54}, \mathrm{a}_{55}, \mathrm{a}_{65}, \mathrm{a}_{66}, \mathrm{P}_{\text {MAX }}$ allowable increase is 0.000 which means that the increase in the value of the variable is fixed and cannot be changed, while the allowable decrease is infinity, which means that the decrease in the value of the variable can change or not remain.

Based on Table 7, the result of the sensitivity analysis with the two-part tariff scheme obtained that the variable $\mathrm{n}, \mathrm{a}_{12}$, $\mathrm{a}_{21}$, $\mathrm{a}_{22}, \mathrm{a}_{32}, \mathrm{a}_{41}, \mathrm{a}_{42}, \mathrm{a}_{51}, \mathrm{a}_{52}, \mathrm{a}_{61}, \mathrm{a}_{62}$, have the allowable increase of 0.000 which means that the increase in the value of the variable is fixed and cannot be changed, while the allowable decrease is infinity which means that the value decreases, this variable may or may not change. In the variable $\mathrm{P}_{X}, \mathrm{X}_{A}, \mathrm{P}_{Y} \mathrm{Y}_{B}$ , the allowable increase is infinity which means that the increase in the value of the variable can be changed or not fixed, while the allowable decrease is 0.000 which means that the decrease in the value of the variable is fixed and cannot be changed. In the variable $\mathrm{a}_{33}, \mathrm{a}_{44}, \mathrm{a}_{45}, \mathrm{a}_{54}, \mathrm{a}_{55}, \mathrm{a}_{65}, \mathrm{a}_{66}, \mathrm{P}_{M A X}$ allowable increase is 0.000 which means that the increase in the value of the variable is fixed and cannot be changed, while the allowable 
Table 9. Recapitulation of Model Solution of Internet Pricing Scheme

\begin{tabular}{|c|c|c|c|c|c|c|c|c|}
\hline \multirow{2}{*}{ Status of Solver } & \multicolumn{2}{|c|}{$\begin{array}{l}\text { C-RAN and Fair } \\
\text { Network Models }\end{array}$} & \multicolumn{2}{|c|}{ Flat-Fee } & \multicolumn{2}{|c|}{$\begin{array}{l}\text { Improved Models } \\
\text { Usage-Based }\end{array}$} & \multicolumn{2}{|c|}{ Two-Part Tariff } \\
\hline & Case 1 & Case 2 & Case 1 & Case 2 & Case 1 & Case 2 & Case 1 & Case 2 \\
\hline Model Class & MINLP & MINLP & NLP & NLP & NLP & NLP & NLP & NLP \\
\hline \multirow{2}{*}{ State } & Local & Local & Local & Local & Local & Local & Local & Local \\
\hline & Optimal & Optimal & Optimal & Optimal & Optimal & Optimal & Optimal & Optimal \\
\hline Objective & 1 & 1 & 1 & 1 & 1 & 1 & 1 & 1 \\
\hline Infeasibility & 0 & 0 & 0 & 0 & 0 & 0 & 0 & 0 \\
\hline Iterations & 163 & 163 & 68 & 70 & 70 & 70 & 70 & 70 \\
\hline \multicolumn{9}{|c|}{ Extended Solver Status } \\
\hline Best Objective & 1 & 1 & 0 & 0 & 0 & 0 & 0 & 0 \\
\hline Update Interval & 2 & 2 & 2 & 2 & 2 & 2 & 2 & 2 \\
\hline GMU (K) & 66 & 66 & 69 & 71 & 69 & 71 & 69 & 69 \\
\hline ER (Sec) & 0 & 0 & 0 & 0 & 0 & 0 & 0 & 1 \\
\hline
\end{tabular}

decrease is infinity, which means that the decrease in the value of the variable can change or not remain.

Based on Table 8 , the result of the sensitivity analysis with the scheme of usage-based obtained that the variable $n, a_{11}, a_{12}$, $\mathrm{a}_{31}, \mathrm{a}_{41}, \mathrm{a}_{42}$, the allowable increase is 0.000 which means that the increase in the value of the variable is fixed and cannot be changed, while the allowable decrease is infinity which means that the value decreases, this variable may or may not change. In the variable $\mathrm{d}_{31}, \mathrm{Y}_{B}$, the allowable increase is infinity which means that the increase in the value of the variable can be changed or not fixed, while the allowable decrease is 0.000 which means that the decrease in the value of the variable is fixed and cannot be changed. In the variable $\mathrm{a}_{33}, \mathrm{a}_{44}, \mathrm{a}_{45}$, $\mathrm{a}_{54}, \mathrm{a}_{55}, \mathrm{a}_{66}$ allowable increase is 0.000 which means that the increase in the value of the variable is fixed and cannot be changed, while the allowable decrease is infinity, which means that the decrease in the value of the variable can change or not remain.

Based on Table 6, Table 7, and Table 8, the results of sensitivity analysis were obtained by each pricing scheme, namely flat-fee, usage-based, and two-part tariff. If the variable is infinity, then the increase or decrease is not fixed and can change, whereas if the variable is 0.000 then the increase or decrease is fixed.

Finally, the recapitulation table of model comparison among the original and improved models are displayed in Table 9 . Based on the results of the recapitulation of the solutions of the C-RAN and Fair Network models with the improved C-RAN model, the fair network and the utility function of CES on the use of traffic data presented in Table 9, the maximum profit for Cases 1 and 2 is the improved C-RAN model in Case 2 with the two-part tariff financing scheme, which is IDR. 1/Kbps. This optimal solution is obtained based on the Extended solver status which shows the largest local optimal value with 83 iterations, which is greater than Case 1. GMU shows the amount of memory allocation used is $69 \mathrm{~K}$ and the length of time used to complete and produce the model is expressed in ER is equal to 1 second.

\section{CONCLUSIONS}

The improved models can be utilized to show the connection between cases by involving CES utility function. By adding the CES utility function to the pricing scheme, Information service providers earn more money than if they only used the initial model. However, obtaining maximum revenue necessitates a number of iterations which results in a huge increase in internet resources. The iteration numbers in case 2 are greater than research previously discussed in (Puspita et al., 2019).

For further investigation, it is also necessary to explore the possibility to increase the number of links in order to create a more realistic network. It also has to do with a software application's ability to run across the numerous variables and parameters involved.

\section{ACKNOWLEDGMENT}

On November 23, 2020, DIPA of the Public Service Agency of Sriwijaya University 2021, SP DIPA-023.17.2.677515/2021, supported the publishing of this article. On April 28, 2021, the Rector's Decree Number: 0010/ UN9/ SK.LP2M.

$\mathrm{PT} / 2021$ was issued.

\section{REFERENCES}

Andersson, J. A. and J. B. Rawlings (2018). Sensitivity Analysis for Nonlinear Programming in CasADi. IFAC-Papers OnLine, 51(20); 331-336

Belghith, A., S. Trabelsi, and B. Cousin (2014). Realistic PerCategory Pricing Schemes for LTE Users. In 12th International Symposium on Modeling and Optimization in Mobile, Ad Hoc, and Wireless Networks (WiOpt), 429-435

Bhattacharya, P. (2017). A Growth Framework Using the Constant Elasticity of Substitution Model. Journal of Applied Mathematics and Physics, 5(11); 2183

Cheng, M. (2019). A Grey CES Production Function Model 
and Its Application in Calculating The Contribution Rate Of Economic Growth Factors. Complexity, 2019(1);1-10.

Cunningham, K. and L. Schrage (2004). The Lingo Algebraic Modeling Language Chapter 6. Applied Optimization: Springer, 159-171

Indrawati, I., S. Erlita, I. Nadeak (2017). Optimasi Model Cloud Radio Access Network (C-RAN) pada Efisiensi Konsumsi Bandwidth dalam Jaringan. Annual Research Seminar, 3(1);117-120

Indrawati, I., Puspita, F. M., Silaen, B. O. M., Yuliza, E., \& Dwipurwani, O. (2020). Selfish User Network Optimization With Celluler Network Traffic Management Model Using Lingo 13.0. Science and Technology Indonesia, 5(2);53-58

Indrawati, I., Puspita, F. M., Resmadona, R., Yuliza, E., Dwipurwani, O., and Octarina, S. (2021). Analysis of Information Service Pricing Scheme Model Based on Customer Self-Selection. Science and Technology Indonesia, 6(4); 34-45

Indrawati, Puspita, F. M., Yuliza, E., Dwipurwani, O., Putri, Y. E., \& Affriyanti. (2019). Improved Cloud Computing Model Of Internet Pricing Schemes Based On Cobb-Douglas Utility Function. Journal of Physics: Conference Series, 1282(1); 012003

Iosifidis, G., L. Gao, J. Huang, and L. Tassiulas (2017). Efficient and Fair Collaborative Mobile Internet Access. IEEE/ACM Transactions on Networking, 25(3); 1386-1400

Li, Y., T. Zhou, Y. Yang, H. Hu, and M. Hamalainen (2016). Fair Downlink Traffic Management for Hybrid LAALTE/Wi-Fi Networks. IEEE Access, 5; 7031-7041

Menth, M. and N. Zeitler (2016). Activity-Based Congestion Management for Fair Bandwidth Sharing In Trusted Packet Networks. IEEE/IFIP Network Operations and Management Symposium, 231-239

Montoya, J., A. Sethi, and N. G. Gómez (2018). A Load-Based And Fair Radio Access Network Selection Strategy with Traffc Offloading In Heterogeneous Networks. In 018 7th International Conference on Computers Communications and Control (ICCCC), 2169-3536

Nurajizah, S., N. A. Ambarwati, and S. Muryani (2020). Sistem Pendukung Keputusan Pemilihan Internet Service Provider Terbaik Dengan Metode Analytical Hierarchy Process. Jurnal Teknologi dan Sistem Informasi, 6(3); 231-238. (in Indonesia)

Peng, M., Y. Li, J. Jiang, J. Li, and C. Wang (2014). Heterogeneous Cloud Radio Access Network; A New Perspective For Enhancing Spectral And Energy Efficiencies. IEEE Wireless Communications, 21(6); 126-135

Puspita, F. M., K. Seman, and B. M. Taib (2015). The Improved Models Of Internet Pricing Scheme Of Multi Service Multi Link Networks With Various Capacity Links. In In Lecture Notes in Electrical Engineering, 315; 851-862
Puspita, F. M., A. Wulandari, E. Yuliza, R. Sitepu, and Y. Yunita (2021). End-to-End Delay QoS Attribute-Based Bundling Strategy of Wireless Improved Reverse Charging Network Pricing Model. Science and Technology Indonesia, 6(1); 30-38

Puspita, F. M., E. Yuliza, O. Dwipurwani, Y. E. Putri, et al. (2019). Improved Cloud Computing Model Of Internet Pricing Schemes Based On Cobb-Douglas Utility Function. In Journal of Physics: Conference Series, 1282;012003

Puspita, F. M., E. Yuliza, W. Herlina, Y. Yunita, and R. Rohania (2020). Improved Multi Service-Reverse Charging Models for the Multi Link Internet wireless Using QOS Bit Error Rate QoS Attribute. Science and Technology Indonesia, 5(1); 6-13

Quaas, M. F., S. Baumgärtner, M. A. Drupp, and J. N. Meya (2020). ntertemporal Utility With Heterogeneous Goods And Constant Elasticity Of Substitution. Economics Letters, 191; 109092

Romdhoni, A. H., M. Wahyuddin, and A. Riyardi (2015). Analisis Fungsi Produksi Frontier Constant Elasticity Substitution Industri Makanan Hingga Pakaian Jadi Di Provinsi Jawa Tengah. University Research Colooquium, ISSN 24079189; 1-15. (in Indonesia)

Sasidhar, T., V. Havisha, S. Koushik, M. Deep, and V. K. Reddy (2016). Load Balancing Techniques for Efficient Traffic Management in Cloud Environment. International Journal of Electrical \& Computer Engineering, 6(3); 963-973

Sitepu, R., F. M. Puspita, E. Kurniadi, S. Apriliyani, et al. (2019). Mixed Integer Nonlinear Programming (MINLP)Based Bandwidth Utility Function On Internet Pricing Scheme With Monitoring And Marginal Cost. International Journal of Electrical \& Computer Engineering, 9(2);1-10

Stechlinski, P., J. Jäschke, and P. I. Barton (2019). Generalized Sensitivity Analysis Of Nonlinear Programs Using A Sequence Of Quadratic Programs. Optimization, 68(2); 485-508

Stechlinski, P., K. A. Khan, and P. I. Barton (2018). Generalized Sensitivity Analysis Of Nonlinear Programs. SIAM Journal on Optimization, 28(1); 272-301

Tsiaflakis, P., Y. Yi, M. Chiang, and M. Moonen (2012). Throughput And Delay Performance Of DSL Broadband Access With Cross-Layer Dynamic Spectrum Management. IEEE Transactions on Communications, 60(9); 2700-2711

Wu, S. Y. and R. Banker (2010). Best Pricing Strategy For Information Services. Journal of the Association for Information Systems, 11(6); 339-336

Wu, H., Liu, L., Zhang, X., \& Ma, H. (2016). Quality Of Video Oriented Pricing Incentive For Mobile Video Offloading. In IEEE INFOCOM 2016 - The 35th Annual IEEE International Conference on Computer Communications, 1-9 